\title{
Síndrome de Caplan: la paradoja del pulmón como origen de la artritis reumatoide
}

\author{
JULIÁN RONDÓN-CARVAJAL* y JAVIER L. GALINDO P.**
}

\section{Caplan syndrome: the paradox of the lung as the origin of rheumatoid arthritis}

Historically, observational field studies have been the starting point for the development of great advances in the understanding of autoimmune diseases. In the case of rheumatoid arthritis (RA), several clinical associations have been described to date which have in common immunological models transverse to its natural history, which has allowed progress in the development of new therapeutic objectives. Our aim is to make a brief description of nodular involvement in RA, based on the clinical observations of Dr. Anthony Caplan in patients at risk of pneumoconiosis, in order to recognize the value of this type of association in professional medical practice.

Key words: Arthritis rheumatoid; Pneumoconiosis; Autoimmune diseases; Interstitial lung diseases; Caplan syndrome.

\section{Resumen}

Históricamente, los estudios de campo observacionales han sido el punto de partida para el desarrollo de grandes avances en el entendimiento de las enfermedades autoinmunes. En el caso de la artritis reumatoide (AR), se han descrito varias asociaciones clínicas hasta la actualidad que tienen en común modelos inmunológicos transversales a la historia natural de la misma, lo que ha permitido avanzar en el desarrollo de nuevos objetivos terapéuticos. Se pretende hacer una breve descripción del compromiso nodular en AR, partiendo de las observaciones clínicas del Dr. Anthony Caplan en pacientes con riesgo de neumoconiosis, con el fin de reconocer el valor de este tipo de asociaciones en el ejercicio médico profesional.

Palabras clave: Artritis reumatoide; Neumoconiosis; Enfermedades autoinmunes; Enfermedades pulmonares intersticiales; Síndrome de Caplan.

\section{Introducción}

La artritis reumatoide (AR) tiene manifestaciones extraarticulares de presentación variable. El pulmón puede verse comprometido en cualquiera de sus compartimentos: parénquima, vía aérea, vasos arteriales o pleura. En el parénquima, puede presentarse enfermedad pulmonar intersticial (EPI) asociada a la AR o a su tratamiento (30$40 \%$ de casos), predominando el patrón de neumonía intersticial usual (NIU) y, en menor medida, neumonía intersticial no específica (NINE).
La presencia de nódulos pulmonares necrobióticos hace parte de otro espectro de presentación clínica. El síndrome de Caplan, que corresponde a la presencia de nódulos reumatoides relacionados a la exposición a carbón o sílice, se presenta en el $0,4 \%$ de los pacientes según la serie original y alcanza una incidencia de $0,75 \%$ en Japón y de $1,5 \%$ en los Estados Unidos ${ }^{1,2}$.

Si bien, la mayoría de casos de EPI y de nódulos reumatoides se presentan en pacientes con AR instaurada, el compromiso intersticial puede preceder el compromiso articular hasta en el 11\%

\footnotetext{
* Médico y cirujano, Universidad de Antioquia. Especialista en Medicina Interna, Pontificia Universidad Javeriana. Médico internista, SURA EPS. Medellín, Colombia.

** Médico y cirujano, Universidad Nacional de Colombia. Especialista en Medicina Interna y Neumología, Universidad Nacional de Colombia. Médico neumólogo, Hospital Universitario Mayor Méderi. Bogotá, Colombia.
} 
de casos, en un promedio de 20 meses $^{3}$. De igual forma, se ha descrito la presencia de nódulos reumatoides en el pulmón hasta en el 3,9\% de pacientes con síndrome de Caplan, antes de la aparición de manifestaciones articulares ${ }^{1,4,5}$. Estos datos sugieren que los orígenes de la AR podrían ubicarse parcialmente en el propio pulmón. La descripción inicial del síndrome de Caplan pudo haber trazado una ruta que recientemente se ha retomado acerca de la fisiopatología de la enfermedad. ¿Acaso es la AR una enfermedad originada por vía inhalatoria?, ¿Podría considerarse una enfermedad profesional?

El objetivo de esta revisión es ilustrar, a través de un caso clínico, algunos aspectos históricos y fisiopatológicos sobre la relación entre AR y silicosis.

\section{Caso clínico}

Hombre de 48 años, natural y procedente de Facatativá, Colombia. Soldador desde hace 30 años. Consultó por cuadro de un año de poliartritis simétrica de predominio en metacarpofalángicas e interfalángicas proximales, así como de codos y rodillas. Refería además pérdida de peso de $11 \mathrm{~kg}$ y disnea progresiva desde hace 3 meses, asociada a episodios de tos con movilización de secreciones. Había sido diagnosticado con AR seropositiva tres años atrás, en manejo con cloroquina, $250 \mathrm{mg}$ /día y prednisolona, $5 \mathrm{mg} /$ día.
Al examen físico, taquicárdico, afebril; con roncus, crépitos en velcro bibasales y tirajes intercostales bajos; sin lesiones en piel o mucosas, ojo rojo u órganomegalias. Al examen osteomuscular, desviación ulnar de ambos carpos y atrofia interósea, sin signos de sinovitis en pequeñas o grandes articulaciones. Limitación para la flexoextensión pasiva de tobillos y codos, sin nódulos subcutáneos.

Los hallazgos de las imágenes fueron compatibles con silicosis conglomerada (Figura 1). Ante la sospecha de tuberculosis pulmonar, dada su asociación con la silicosis, se tomaron baciloscopias de esputo que fueron negativas. Se realizó fibrobroncoscopia con lavado broncoalveolar, con hallazgos de endobronquitis purulenta. Las tinciones, los cultivos y el GeneXpert en el lavado fueron negativos. El test de ELISA para VIH fue no reactivo.

Durante la internación, presentó sinovitis en rodilla derecha y tobillo izquierdo con elevación de reactantes de fase aguda, puntuando para actividad moderada de la AR (DAS28 entre 3,2-5,1). El caso fue llevado a junta médica, considerándose realizar biopsia pulmonar abierta o percutánea de las pseudomasas, pero por el alto riesgo de neumotórax no se realizó. Teniendo en cuenta la caquexia y los hallazgos imagenológicos, se decidió dar inicio a terapia tetraconjugada para tuberculosis pulmonar, a pesar de los estudios microbiológicos negativos.

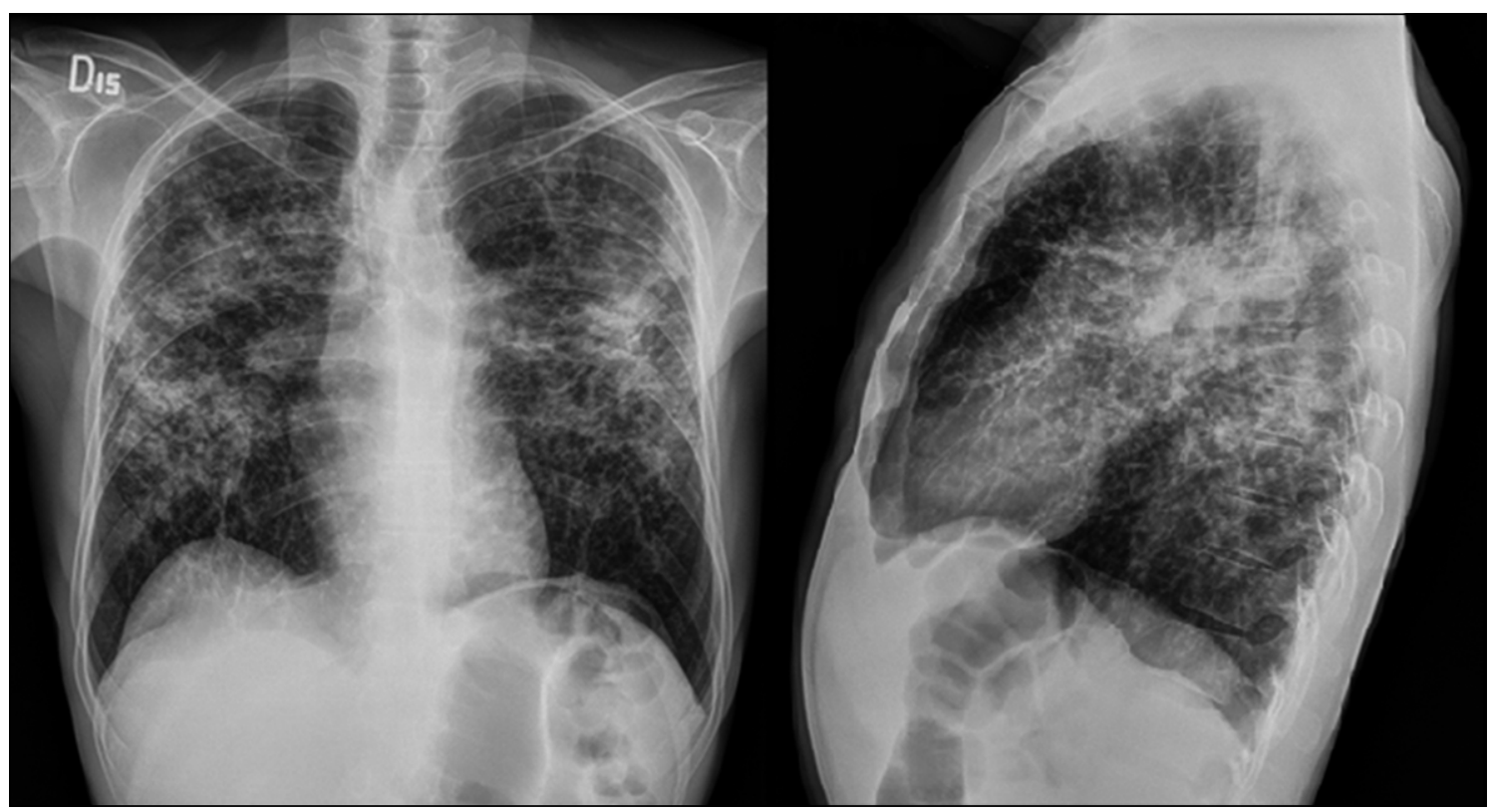

Figura 1. Radiografía de tórax, proyección AP y lateral. Patrón micronodular difuso de distribución aleatoria, de predominio en los lóbulos superiores y regiones parahiliares, donde confluyen formado masas. Silueta cardíaca y mediastino sin alteraciones. 


\section{Aspectos clínicos: perspectiva histórica}

La asociación entre la AR y las enfermedades pulmonares ocupacionales relacionadas con la exposición crónica a polvos minerales, como el carbón o la sílice, fue publicada hacia 1953 por el doctor Anthony Caplan ${ }^{1}$, quien describió entre 14 mil trabajadores de minas de carbón en Cardiff (sur de Gales) 51 casos con diagnóstico confirmado de AR, de los cuales casi el 90\% presentaban hallazgos de fibrosis pulmonar masiva, en comparación con el 30\% de pacientes sin diagnóstico de AR; 13 de estos 51 (25\%) casos presentaban un patrón radiológico particular, consistente en múltiples nódulos pulmonares entre 0,5 y $5 \mathrm{~cm}$ de diámetro, de rápida aparición, localizados hacia la periferia de ambos ápices con opacidades intersticiales de extensión variable, no compatibles con fibrosis pulmonar masiva (trasfondo de neumoconiosis simple en cerca de $45 \%$ de casos). Su patología era similar a la de los nódulos reumatoides: capa de células histiocitarias en empalizada de contenido silicótico y acúmulos de polimorfonucleares neutrófilos rodeando un centro necrótico con depósitos variables de colágeno suelto en capas concéntricas, como lo describió Gough en $1955^{6}$.

Esta presentación nodular se denominó inicialmente neumoconiosis reumatoide ${ }^{6,7}$; Honma $y$ Vallyathan la clasificaron en su momento en dos tipos: clásica (síndrome de Caplan), con nódulos reumatoides grandes como lo describió Caplan entre 1953 y 1959 y por Gough en 1955, y silicótica, con micronódulos silicóticos apicales acompañado de $\mathrm{AR}^{2}$.

Neumoconiosis es el término utilizado para las enfermedades asociadas con la inhalación de polvos minerales, muchos de los cuales se encuentran en el ambiente; sin embargo, la mayoría ocurren ante exposiciones de dimensiones industriales, siendo silicosis la entidad más representativa. El principal factor de riesgo para su desarrollo es el grado de exposición al polvo mineral (para carbón, trazado sobre un límite superior de mandato federal de $\left.2 \mathrm{mg} / \mathrm{m}^{3}\right)^{8,9,10}$. Al sobrepasarse los mecanismos de depuración pulmonar, macrófagos alveolares cargados con partículas extracelulares se acumulan en el intersticio, hacia las regiones peribroncovasculares, con posterior participación de neumocitos tipo 1 , formación de fibras de reticulina entre estos y eventual proliferación de colágeno ${ }^{8}$.

Las observaciones iniciales de Caplan en mineros con diagnóstico confirmado de AR fueron descritas como "numerosas opacidades redondeadas dispersas difusamente en ambos parénquimas pulmonares, de rápida progresión, dispuestas en patrón parcheado de similar tamano y tasa de crecimiento, con pocos cambios en su configuración tras pocos años de seguimiento"; así mismo, estas nuevas opacidades se presentaban sobre hallazgos por imagen compatibles con neumoconiosis grado 1: mínimas opacidades intersticiales, sin parecerse al patrón intersticial descrito en pacientes con AR en 1948, por Ellman y Ball ${ }^{11}$.

\section{Neumoconiosis y tuberculosis pulmonar}

La asociación entre silicosis y tuberculosis pulmonar ha sido bien establecida. Las micobacterias pueden encapsularse en los nódulos silicóticos, llevando incluso a cavitación en ausencia de infección activa. En el estudio de Caplan ${ }^{1}, 6$ de 51 casos con diagnóstico de AR tenían opacidades radiológicas indistinguibles de tuberculosis pulmonar; adicionalmente, $20 \%$ del total de pacientes tenían opacidades apicales sugestivas de tuberculosis pulmonar inactiva y solo 4 tenían un patrón de neumoconiosis simple. Pese a lo anterior, se estudiaron muestras de esputo para baciloscopia y cultivo en 23 pacientes seleccionados al azar, todas negativas; uno de los casos tuvo aislamiento de $M$. tuberculosis en 2 de 7 lavados gástricos, desarrollando a los 9 meses antes del debut de la AR una pequeña opacidad irregular en ápice derecho, que progresó en tamaño a los 4 meses de seguimiento ${ }^{1,11}$.

La silicosis está asociada a defectos en la inmunidad celular efectiva. Por lo anterior, todos los pacientes con silicosis deben ser tamizados para tuberculosis latente con tuberculina (PPD) o pruebas de liberación de interferón-gamma $(\text { IGRA })^{8,12}$.

\section{Neumoconiosis, ¿más allá del pulmón?}

En el siglo XVI, Georg Bauer, médico sajón, describió en su famoso tratado De re metallica (1556) la diversidad de enfermedades padecidas por los trabajadores mineros: «algunas -escribia-afectan a las articulaciones, otras atacan a los pulmones, algunas a los ojos, y, finalmente, algunas son fatales para los hombres. [...] En las minas donde el agua es abundante y muy fría, daña con frecuencia las extremidades, pues el frío es perjudicial para los nervios. Por otro lado, algunas minas son tan secas que están totalmente desprovistas de agua, y la sequedad hace mayor daño aún en los trabajadores, pues 
el polvo que es desprendido en la excavación penetra en la tráquea y en los pulmones, y produce dificultades en la respiración [...] Si el polvo tiene propiedades corrosivas, carcome los pulmones $y$ consume el cuerpo. $\rangle^{13}$

Una década más tarde se publicó la obra póstuma de Paracelso, Von der Bergsucht und Anderen Bergkrankheiten -De los oficios y enfermedades de la montaña- (1567) en la que se señala la relación existente entre minería y enfermedades pulmonares ${ }^{14}$.

A comienzos del XVIII, Bernardino Ramazzini en su obra De morbis artificum diatriba -Tratado de las enfermedades de los artesanos- (1700) dedicaba el primer capítulo a las causadas por la actividad minera, asociando los siguientes signos y síntomas: "disnea, tisis, apoplejía, parálisis, caquexia, tumores en los pies, pérdida de dientes, úlceras en las encías, dolores y temblores en las

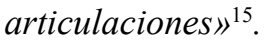

Teniendo en cuenta lo anterior, ¿podríamos suponer que el pulmón es el órgano donde se gestan los mecanismos prima facie de una enfermedad sistémica como la AR?

\section{Fisiopatología}

En la AR se han descrito polimorfismos en el complejo mayor de histocompatibilidad tipo II $\mathrm{y}$ en los genes PTPN22 (proteína tirosina fosfatasa) y PADI4 (peptidilarginina deiminasa) que aumentan el riesgo de presentar la enfermedad de 2 a 5 veces. La interacción entre estos, modificaciones epigenéticas y exposiciones ambientales conducen a pérdida de tolerancia inmune con la manifestación fenotípica de inflamación sinovial crónica o de daño pulmonar ${ }^{16,17}$.

Proteínas de la membrana sinovial, como la fibrina o la vimentina, al citrulinarse se convierten en inmunógenos, que generan una respuesta autoinmune mediada por linfocitos B y probablemente por linfocitos $\mathrm{T}$ contra estos péptidos citrulinados $^{18,19}$. Estas proteínas se unen al HLADRB1 en las células presentadoras de antígenos favoreciendo la producción de anticuerpos antipéptidos cíclicos citrulinados (anti-CCP) (Figura $2)^{19}$. La identificación de anti-CCP no implica el desarrollo de enfermedad; sin embargo, pueden preceder 3-5 años la manifestación articular; entre 21,5 a $46,0 \%$ de estos casos evolucionarán a AR.

En el proceso inflamatorio local, algunas citoquinas proinflamatorias, como el factor de necrosis tumoral- $\alpha$ (TNF- $\alpha$ ) y la IL-1 inducen la liberación de metaloproteinasas y otros mediadores como el RANK-L. ${ }^{18}$, que resulta en destrucción de matriz extracelular en hueso y cartílago sinovial.

La exposición inhalatoria a humo de cigarrillo, polvo de sílice o carbón, infecciones por Mycoplasma spp o virus de Epstein-Barr, parecen estimular la citrulinización de algunas proteínas a nivel postrasduccional (conversión de residuos de arginina en citrulina) mediada por la enzima PADI4, con la activación simultánea de los macrófagos $^{18,20}$.

La relación entre neumoconiosis (especialmente, silicosis) y el desarrollo de trastornos autoinmunes se ha reconocido desde 1950, con numerosos reportes de neumoconiosis asociada a esclerosis sistémica (síndrome de Erasmus),

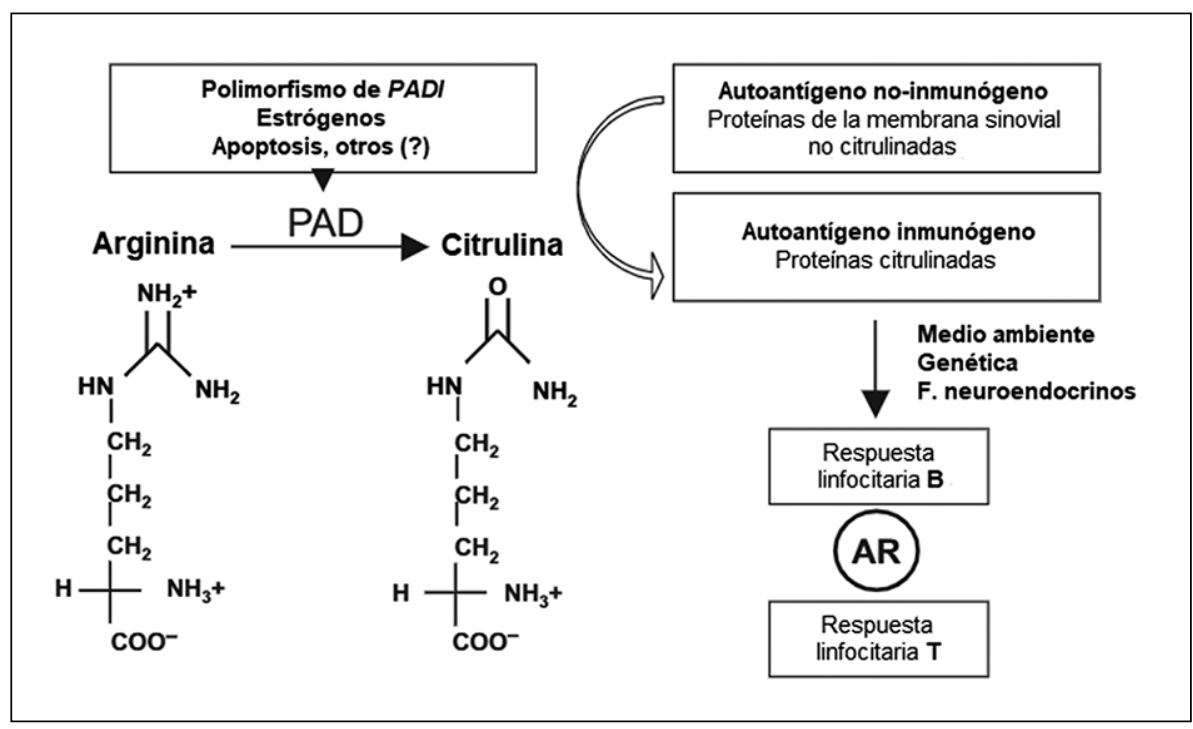

Figura 2. Citrulinización de proteínas en el desarrollo de AR. Adaptado de Correa et al. ${ }^{19}$. 
lupus eritematoso sistémico y enfermedad mixta del tejido conectivo ${ }^{21-24}$. Estudios experimentales demuestran que la sílice desencadena un efecto adyuvante en la respuesta inmune de los animales, estimulando monocitos y macrófagos, con la consiguiente liberación de IL-1, factor estimulante de colonias de granulocitos y macrófagos y TNF- $\alpha$, lo que lleva a cambios silicóticos en pulmón $^{25,26}$.

\section{De la neumoconiosis como modelo de autoinmunidad en AR}

Tras la activación de la inmunidad innata y posterior estimulación de la inmunidad adaptativa, puede haber pérdida gradual de la tolerancia que se ha relacionado con expresiones extrapulmonares de autoinmunidad en pacientes genéticamente predispuestos ${ }^{27,28}$. Existe evidencia de que la exposición a la sílice cristalina contribuye al desarrollo de enfermedades autoinmunes, incluyendo LES, AR, esclerosis sistémica y vasculitis asociada anticuerpos contra el citoplasma del neutrófilo (ANCA) ${ }^{28}$.

En el caso de la sílice $\left(\mathrm{SiO}_{2}\right)$ u óxido de silicio, dispuesto en la naturaleza como cuarzo, la respuesta inflamatoria está mediada por el inflamasoma NLRP3 (Figura 3), modulado a su vez por IL-1 $1 \beta^{29,30}$, a partir de receptores "scavenger", lo que lleva a ruptura lisosómica y liberación de catepsina $\mathrm{B}$, con generación de especies reactivas de oxígeno (ROS) y flujo de salida de potasio ${ }^{31}$. El predominio de una respuesta Th2 precipitada por IL-10 favorece la fibrosis pulmonar, al aumentar niveles de IL-4 y IL-13, citocinas implicadas en vías alternas del TGF- $\beta^{32}$; así pues, se presume que los fenómenos inflamatorios son diferentes a los procesos fibrogénicos en pulmón.

Entre 47 y $77 \%$ de individuos expuestos a sílice desarrollan silicosis; entre estos, se describe hipergamaglobulinemia en $65 \%$ de casos y ANAS en $34 \%$ o más, hallazgos serológicos que parecen generarse en pulmón a través de la inmunidad innata con generación de clonas de linfocitos autorreactivos y daño tisular se-

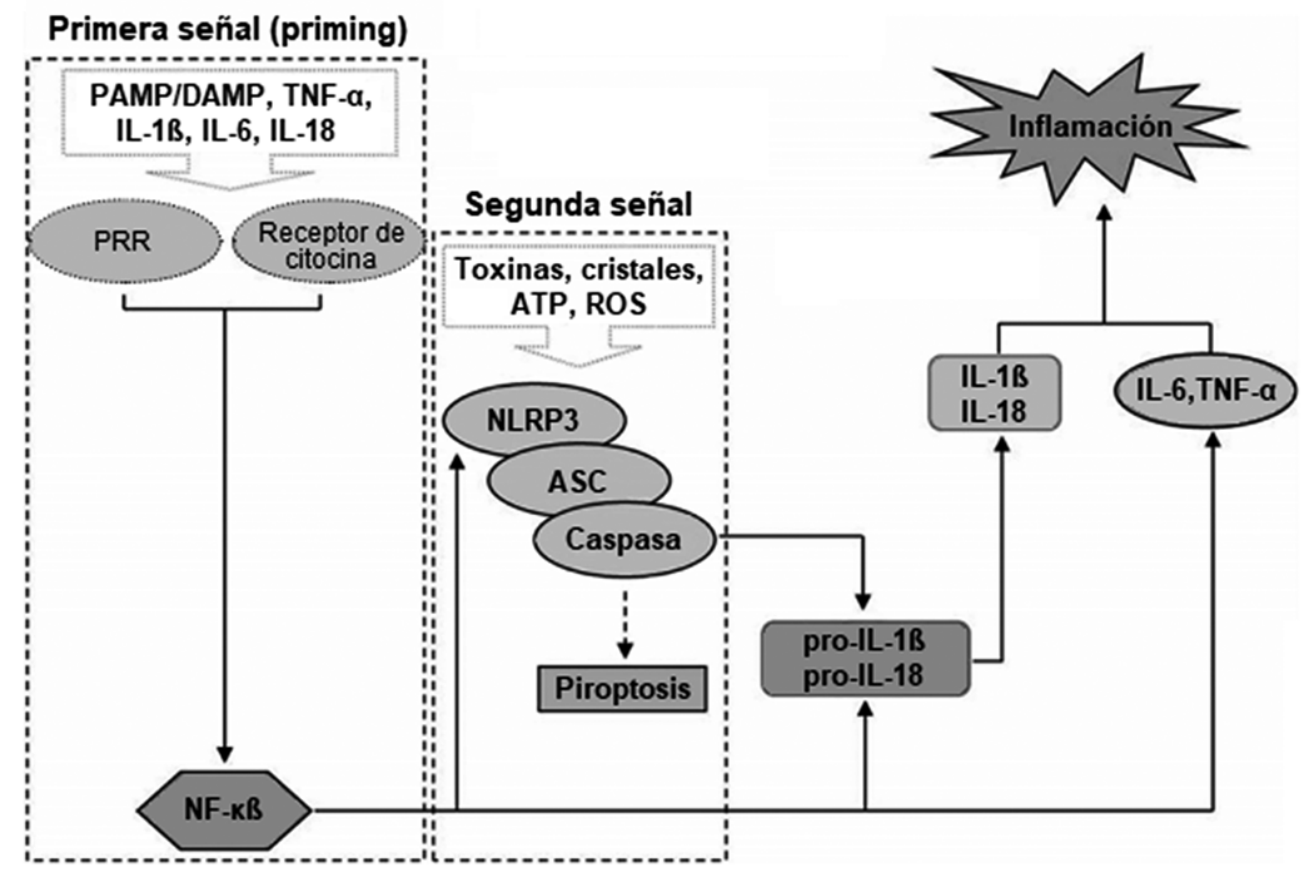

Figura 3. Activación del inflamasoma NLRP3. Patrones moleculares asociados a patógenos (PAMP) o peligro (DAMP) así como citocinas proinflamatorias (TNF- $\alpha$, IL-1ß, IL-6, IL-18) estimulan la activación de NF- $\kappa \beta$ a través de sus respectivos receptores. Esto promueve la síntesis de NLRP3, pro-IL-1ß y pro-IL-18, y citocinas proinflamatorias (IL-6, IL-8 y TNF- $\alpha$, entre otras). La activación de la segunda señal permite el ensamblaje del inflamasoma NLRP3 o la formación del complejo NLRP3/ASC/Pro-caspasa-1, para la activación de la caspasa-1 y la maduración proteolítica de pro-IL-1ß y pro-IL-18, que da origen a las formas biológicamente activas de IL-1ß e IL-18 y citocinas proinflamatorias. (Adaptado de Hernández-López JC et al. $\left.{ }^{51}\right)$. 
cundario; al parecer, los autoanticuerpos van dirigidos a productos derivados de la apoptosis de macrófagos alveolares inducida por el material inorgánico acumulado, con participación de IFN-a/ß e IFN gama ${ }^{35,36}$.

Se propone que la ausencia de receptores "scavenger" favorecen la autoinmunidad. De igual forma, parece que una regulación negativa en células Th17 reguladoras perpetúa la producción de autoanticuerpos en el contexto de exposición repetida a la sílice ${ }^{37,38}$.

Finalmente, se ha venido postulando que la EPI asociada a AR tiene origen en el pulmón, donde se citrulinizan proteínas, producto de los procesos inflamatorios. Klareskog et al. ${ }^{39}$ observaron una relación entre HLA-DRB1*0401 y anticuerpos anti-CCP en individuos fumadores con AR. El riesgo relativo de desarrollar AR y la presencia de anticuerpos antiCCP positivos es 20 veces mayor para los pacientes fumadores con este alelo ${ }^{39,40}$.

La asociación entre la citrulinación de proteínas en los pulmones de fumadores y el inicio de la respuesta inmune contra dichas proteínas en $\mathrm{AR}$ es un fenómeno que podría ser extrapolado a pacientes con neumoconiosis (sílice, carbón y asbesto), en quienes la exposición a largo plazo pudiese llevar a liberación de la enzima peptidil arginina deiminasa (PAD), favoreciendo la citrulinación de proteínas liberadas por el daño pulmonar directo y el potencial desarrollo de autoinmunidad, como se muestra a continuación ${ }^{41,42}$ (Figura 4).

\section{Consideraciones terapéuticas}

Pirfenidona y nintedanib, son medicamentos que han demostrado disminuir la progresión de la fibrosis pulmonar idiopática (FPI), la EPI fibrosante de peor pronóstico ${ }^{43,44}$. Pirfenidona es un medicamento con múltiples mecanismos de acción, dentro de los que se incluye la inhibición de TGF- $\beta$, TNF- $\alpha$ y factor de crecimiento derivado de plaquetas (PDGF); nintedanib es un inhibidor de tirosina quinasa que actúa sobre el sitio de unión del ATP del receptor del factor de crecimiento vascular endotelial (VEGFR) 1,2 y 3 , del receptor del factor de crecimiento fibroblástico 1,2 y 3 y del receptor del PDGF $\alpha$ y $\beta$. Ambos agentes interfieren vías de señalización relacionadas con proliferación, migración y diferenciación de fibroblastos en intersticio pulmonar ${ }^{45,46}$.

Algunas de EPI crónicas, incluyendo silicosis y secundarias a autoinmunidad, pueden tener un curso progresivo con deterioro de la función pulmonar asimilable al de FPI. Esta presentación clínica conoce como "fenotipo fibrosante progresivo" 47 , caracterizado por un incremento de la extensión de fibrosis en tomografía de tórax, declinación de función pulmonar y empeoramiento de los síntomas.

El tratamiento de la EPI relacionada a exposiciones ocupacionales inicia por evitar la exposición al agente causal; no existen intervenciones farmacológicas que curen la fibrosis. Para EPI asociada a AR, habitualmente los esteroides en combinación con otros agentes inmunosupresores

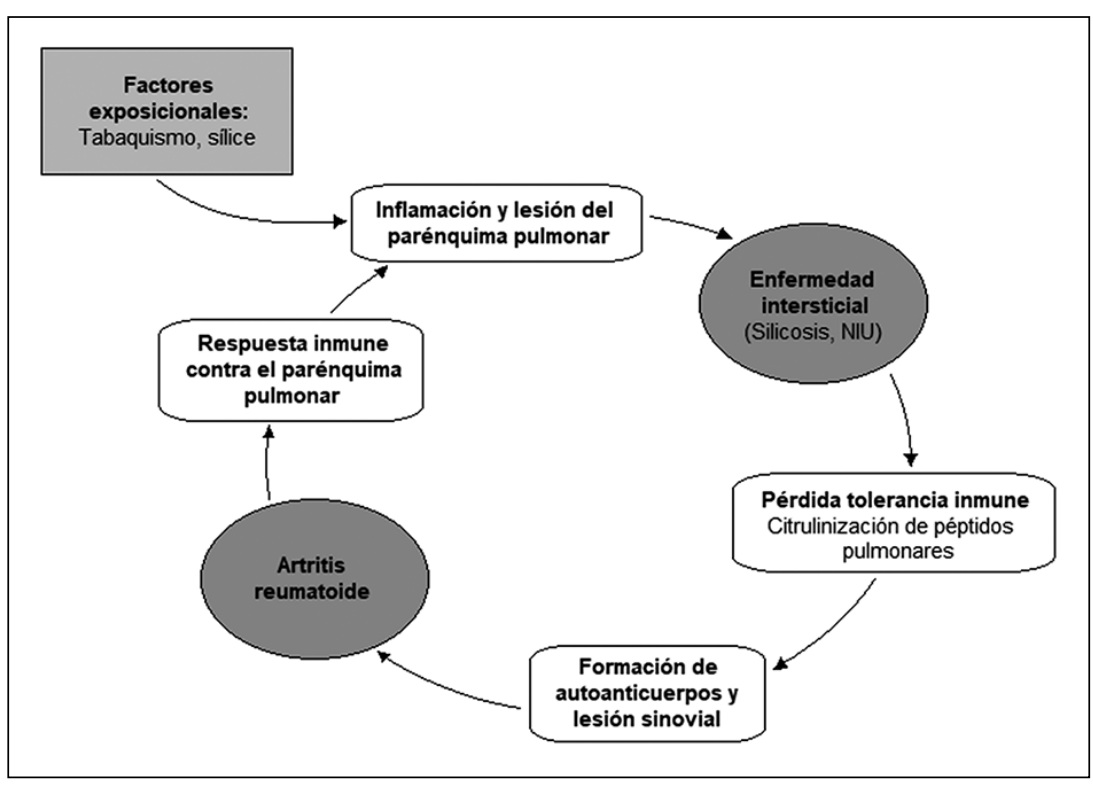

Figura 4. Modelo de correlación fisiopatológica entre neumoconiosis y AR (Figura elaborada por los autores). 
son usados, pero su eficacia es extrapolada de estudios en esclerosis sistémica y estudios observacionales Algunos de los medicamentos usados para el compromiso articular, como metotrexato, leflunomida y medicamentos biológicos, podrían exacerbar la EPI preexistente ${ }^{48}$.

El estudio INBUILD evaluó nintedanib contra placebo en 663 pacientes adultos con EPI fibrosante no FPI con fibrosis $>10 \%$ en tomografía de tórax, capacidad vital forzada $(\mathrm{CVF}) \geq 45 \%$ del predicho y capacidad de difusión de monóxido de carbono de 30 a $<80 \%$ del predicho. Para ser incluidos, estos pacientes debían presentar en los últimos 24 meses enfermedad progresiva: disminución en $\mathrm{CVF} \geq 10 \%$, disminución en $\mathrm{CVF}$ de 5 a $<10 \%$ con empeoramiento de síntomas respiratorios, disminución en CVF de $5 \mathrm{a}<10 \%$ con extensión de la fibrosis en la TC de tórax o empeoramiento de síntomas respiratorios con extensión de fibrosis en tomografía de tórax ${ }^{49}$. De los casos de EPI fibrosante incluidos, 13\% tenían EPI asociada a AR y $12,2 \%$ causas misceláneas de fibrosis, incluyendo neumoconiosis no especificadas ${ }^{50}$.

El objetivo primario del estudio fue evaluar tasa de declinación anual de la CVF durante 52 semanas. La tasa ajustada de deterioro de la CVF fue de $-80,8 \mathrm{ml} /$ año con nintedanib vs. $-187,8 \mathrm{ml} /$ año con placebo, teniendo diferencia entre grupos de $107 \mathrm{ml} /$ año (IC95\% de 65,4 a 148,5; $p<$ $0,001)$. El efecto de nintedanib fue independiente del patrón de daño tomográfico. No hubo repercusión del uso de nintedanib en desenlaces secundarios como calidad de vida, exacerbaciones y mortalidad. El efecto de nintedanib en reducción de tasa de disminución de la CVF fue consistente en todos los subtipos de EPI incluidas abriendo la posibilidad de su uso en EPI con "fenotipo fibrosante progresivo".

Estudios con pirfenidona y otras terapias dirigidas a tratar la fibrosis pulmonar se encuentran actualmente en curso. Aún falta evidencia sólida respecto al tratamiento en estos escenarios clínicos puntuales.

\section{Conclusiones}

La presencia de proteínas citrulinadas generadas por exposición pulmonar prolongada a noxas ambientales persistentes como minerales inorgánicos en el contexto de las neumoconiosis pueden favorecer el desarrollo de AR en un huésped con características genéticas particulares.

Los autoantígenos citrulinados que muestran mayor especificidad para AR son fibrinógeno, vi- mentina, colágeno tipo II y enolasa; estos pudiesen ser productos de degradación de procesos de fibrogénesis y apoptosis inducida en poblaciones de macrófagos alveolares.

De igual forma, las isoformas PAD2 y PAD4 de la enzima peptidil arginina deiminasa, se asocian con la generación de estos péptidos citrulinados, considerándose que en casos de antecedentes laborales relacionados con minería (principalmente, relacionados a la sílice) el pulmón, más que afectado en segunda instancia, pudiese llegar a ser actor primario en el desarrollo de la AR, como en su momento lo alcanzó a deducir el doctor Anthony Caplan, a partir de sus observaciones clínicas, realizadas hace más de 50 años.

La evidencia científica para el uso de agentes antifibróticos (nintedanib, pirfenidona) en el tratamiento de las EPI fibrosantes progresivas no FPI, incluyendo las asociadas a AR o a neumoconiosis, está en construcción. Ninguno de estos medicamentos tiene a la fecha licencia para su prescripción. En particular, para el caso de neumoconiosis asociada a AR (síndrome de $\mathrm{Ca}$ plan), la recomendación general es evitar la exposición al agente ambiental y optimizar terapia con DMARD (Disease-modifying antirheumatic drugs), asociada o no a esteroide.

\section{Bibliografía}

1.- CAPLAN A. Certain unusual radiological appearances in the chest of coal-miners suffering from rheumatoid arthritis. Thorax 1953 ;8: 29-37.

2.- MELLO DE CAPITANI E, SCHWELLER M, MENDES DA SILVA C, METZE K, FIGUEIRAS PEDREIRA DE CERQUEIRA EM, BARROS BÉRTOLO M. Rheumatoid pneumoconiosis (Caplan's syndrome) with a classical presentation. J Bras Pneumol 2009; 35: 942-6.

3.- WALKER WC, WRIGHT V. Pulmonary Lesions and Rheumatoid Arthritis. Medicine; 1968; 47: 501-20. URL.

4.- ANTIN-OZERKIS D, EVANS J, RUBINOWITZ A, HOMER RJ, MATTHAY RA. Pulmonary manifestations of rheumatoid arthritis. Clin Chest Med 2010; 31: 451-78.

5.- MEGAN SHAW M, COLLINS BF, HO LA, RAGHU G. Rheumatoid arthritis-associated lung disease. Eur Respir Rev 2015; 24: 1-16. DOI: 10.1183/09059180.00008014.

6.- GOUGH J, RIVERS D, SEAL RME. Pathological studies of modified pneumoconiosis in coal-miners with rheumatoid arthritis; Caplan's syndrome. Thorax 1955; 10: 9-18.

7.- ROZENBERG D, SHAPERA S. What to do with all of these lung nodules? Can Respir J 2014; 21: e52-e4. 
8.- KARKHANIS VS, JOSHI JM. PNEUMOCONIOSES. Indian J Chest Dis Allied Sci 2013; 55: 25-34.

9.- SHAH SH, NAGYMANYOKI Z, RAMAIYA NH, HOWARD S. AIRP best cases in radiologic-pathologic correlation: coal workers' pneumoconiosis. Radiographics 2012; 32: 2047-52. doi:10.1148/rg.327115137.

10.- Fourth International Pneumoconiosis Conference. Report of the working party on the Definition of Pneumoconiosis. Geneva: International Labour Organization; 1971.

11.- MIALL WE, CAPLAN A, COCHRANE AL, KILPATRICK GS, OLDHAM PD. An epidemiological study of rheumatoid arthritis associated with characteristic chest X-ray appearances in coal-workers. Br Med J 1953; 2 (4848): 1231-6. doi:10.1136/bmj.2.4848.1231.

12.- BARBOZA CE, WINTER DH, SEISCENTO M, SANTOS U DE P, TERRA FILHO M. Tuberculosis and silicosis: epidemiology, diagnosis and chemoprophylaxis. J Bras Pneumol 2008; 34: 959-66.

13.- Georgius Agricola, De Re Metallica, trans. por Herbert Clark Hoover y Lou Henry Hoover, Londres, The mining magazine. Salisbury House, London, E.C., 1912, libro VI, p. 214.

14.- NAVA HERNÁNDEZ R. "Antecedentes históricos de la salud en el trabajo", en C.M. Barquín et al. Sociomedicina, México, Méndez Editores, 1994, p. 533.

15.- RAMAZZINI B. Tratado sobre las enfermedades de los trabajadores: traducción comentada de la obra 'De morbis artificum diatriba' de Bernardino Ramazzini, s. XVIII, Madrid, Instituto Nacional de Seguridad e Higiene en el Trabajo, Asociación Instituto Técnico de Prevención, 2012, p. 16.

16.- FIRESTEIN GS. Etiology and Pathogenesis of Rheumatoid Arthritis. 9th ed. Kelley's Textbook of Rheumatology 2013; 1059-108.

17.- SCOTT DL F, WOLFE F, HUIZINGA TW. Rheumatoid arthritis. Lancet 2010; 376 (9746): 1094-108.

18.- MCINNES IB, SCHETT G. The pathogenesis of rheumatoid arthritis. N Engl J Med 2011; 365 (23): 2205-19.

19.- CORREA PA, TOBÓN GJ, CITERA G, CADENA J, SCHNEEBERGER E, CAMARGO JF, MALDONADO-COCCO JA, ANAYA JM. Anticuerpos anti-CCP en artritis reumatoidea: relación con características clínicas, citocinas Th1/Th2 y HLA-DRB1. Biomédica 2004; 24 (2): 140-52. [ citado 'en línea' 03.05.2020].

20.- KLARESKOG, L, CATRINA AI, PAGET S. Rheumatoid arthritis. Lancet 2009; 373 (9664): 659-72.

21.- MACHADO DE MIRANDA AA, CARVALHO NASCIMENTO A, LIMA PEIXOTO I, ALVES SCRIGNOLI J, DE LUCENA CARDOSO MS, EUZÉBIO RIBEIRO SL. Erasmus syndrome-silicosis and systemic sclerosis. Rev Bras Reumatol 2013; 53 :31013.

22.- SPINELLI DA SILVA TELES JA. Silicosis and systemic sclerosis (Erasmus syndrome): the importance of the recognition of the association between diseases. Rev
Bras Med Trab 2016; 14 (1): 45-51.

23.- LUCAS CD, AMFT N, REID PT. Systemic lupus erythematosus complicating simple silicosis. Occupational Medicine 2014; 64: 387-90.

24.- SÁNCHEZ-ROMAN J, WICHMANN I, SALABERRI J, VARELA JM, NUÑEZ-ROLDAN A. Multiple clinical and biological autoimmune manifestations in 50 workers after occupational exposure to silica. Ann Rheum Dis 1993; 52: 534-8.

25.- MARCUS DM. An analytical review of silicone immunology. Arthritis \& rheumatism 1996; 39 (10): 1619-26.

26.- SEN S, MITRA R, MUKHERJEE S, DAS PK, MOITRA S. Silicosis in Current Scenario: A Review of Literature. Current Respiratory Medicine Reviews 2016; 12: 56-64.

27.- JAYAPAL M, BHATTACHARJEE RN, MELENDEZ AJ, HANDE MP. Environmental toxicogenomics: a post-genomic approach to analyzing biological responses to environmental toxins. Int J Biochem Cell Biol (2010) 42:230-40. doi:10.1016/j.biocel.2009.10.007.

28.- MILLER FW, ALFREDSSON L, COSTENBADER KH, KAMEN DL, NELSON LM, NORRIS JM, et al. Epidemiology of environmental exposures and human autoimmune diseases: findings from a National Institute of Environmental Health Sciences Expert Panel Workshop. J Autoimmun 2012; 39: 259-71. doi:10.1016/j. jaut.2012.05.002.

29.- LEUNG CC, YU IT, CHEN W. Silicosis. Lancet 2012; 379: 2008-18. doi:10.1016/S0140-6736(12)60235.

30.- CASSEL SL, EISENBARTH SC, IYER SS, SADLER JJ, COLEGIO OR, TEPHLY LA, et al. Th Nalp3 inflammasome is essential for the development of silicosis. Proc Natl Acad Sci U S A 2008; 105: 9035-40. doi:10.1073/pnas.0803933105.

31.- HORNUNG V, BAUERNFEIND F, HALLE A, SAMSTAD EO, KONO H, ROCK KL, et al. Silica crystals and aluminum salts activate the NALP3 inflammasome through phagosomal destabilization. Nat Immunol 2008; 9: 847-56. doi:10.1038/ni.1631.

32.- BARBARIN V, XING Z, DELOS M, LISON D, HUAUX F. Pulmonary overexpression of IL-10 augments lung fibrosis and $\mathrm{Th} 2$ responses induced by silica particles. Am J Physiol Lung Cell Mol Physiol 2005; 288: L841-8. doi:10.1152/ajplung.00329.2004.

33.- PFAU JC, SERVE KM, NOONAN CW. Autoimmunity and asbestos exposure. Autoimmune Dis 2014. 2014: 782045. doi:10.1155/2014/782045.

34.- CONRAD K, MEHLHORN J, LUTHKE K, DORNER T, FRANK KH. Systemic lupus erythematosus after heavy exposure to quartz dust in uranium mines: clinical and serological characteristics. Lupus 1996; 5 : 62-9.

35.- STEENLAND K. One agent, many diseases: exposureresponse data and comparative risks of different outcomes following silica exposure. Am J Ind Med 2005; 48 : 16-23. doi:10.1002/ajim.20181. 
36.- DOLL NJ, STANKUS RP, HUGHES J, WEILL H, GUPTA RC, RODRIGUEZ M, et al. Immune complexes and autoantibodies in silicosis. J Allergy Clin Immunol 1981; 68: 281-5. doi:10.1016/0091-6749(81)901524.

37.- WERMELING F, CHEN Y, PIKKARAINEN T, SCHEYNIUS A, WINQVIST O, IZUI S, et al. Class A scavenger receptors regulate tolerance against apoptotic cells, and autoantibodies against these receptors are predictive of systemic lupus. J Exp Med 2007; 204: 2259-65. doi:10.1084/jem.20070600.

38.- MICHELS-VAN AMELSFORT JM, WALTER GJ, TAAMS LS. CD4+CD25+ regulatory $\mathrm{T}$ cells in systemic sclerosis and other rheumatic diseases. Expert Rev Clin Immunol 2011; 7: 499-514. doi:10.1586/eci.11.28.

39.- KLARESKOG L, STOLT P, LUNDBERG K, KÄLLBERG H, BENGSTON C, GRUNEWALD J, et al. A new model for etiology of rheumatoid arthritis: smoking may tigger HLA-DR (shared epitope)- restricted immune reactions to antigens modified by citrullination. Arhritis Rheum 2006; 54: 38-46.

40.- HILL JA, BELL DA, BRINTNELL W, YUE D, WEHLRLI B, JEVNIKAR AM, et al. Artrhritis induced by posttranslationally modified (citrullinated) fibrinogen in DR4-IE transgenic mice. J Exp Med 2008; 205: 967-79.

41.- MAKRYGIANNAKIS D, HERMANSSON M, ULFGREN AK, NICHOLAS AP, ZENDMAN AJ, EKLUND A, et al. Smoking increases peptidylarginine deiminase 2 enzyme expression in human lungs and increases citrullination in BAL cells. Ann Rheum Dis 2008; 10: 826-50.

42.- KLARESKOG L, RÖNNELID J, LUNDBERG $\mathrm{K}$, PADYUKOV L, ALFREDSSON L. Immune to Citrullinated Proteins in rheumatoid arthritis. Ann Rev Immunol 2008; 26: 651-75.

43.- KING TE JR, BRADFORD WZ, CASTROBERNARDINI S, FAGAN EA, GLASPOLE I, GLASSBERG MK, et al. A phase 3 trial of pirfenidone in patients with idiopathic pulmonary fibrosis. N Engl J Med 2014; 370 (22): 2083-92. doi: 10.1056/NEJMoa1402582.

44.- RICHELDI L, DU BOIS RM, RAGHU G, AZUMA A, BROWN KK, COSTABE LU, et al. Efficacy and safety of nintedanib in idiopathic pulmonary fibrosis. N Engl J Med. 2014;370(22):2071-82. doi: 10.1056/ NEJMoa1402584.

45.- XAUBET A, SERRANO-MOLLAR A, ANCOCHEA J. Pirfenidone for the treatment of idiopathic pulmonary fibrosis. Expert Opin Pharmacother 2014; 15: 275-81. doi: $10.1517 / 14656566.2014 .867328$.

46.- WOLLIN L, DISTLER JHW, REDENTE EF, RICHES DWH, STOWASSER S, SCHLENKERHERCEG $R$, et al. Potential of nintedanib in treatment of progressive fibrosing interstitial lung diseases. Eur Respir J 2019; 54 (3): 1900161. doi: 10.1183/13993003.00161-2019.

47.- WELLS AU, BROWN KK, FLAHERTY KR, KOLB $\mathrm{M}$, THANNICKAL VJ. What's in a name? that which we call IPF, by Any Other name would act the same. Eur Respir J 2018 May 17; 51 (5). pii: 1800692. doi: 10.1183/13993003.00692-2018.

48.- RICHELDI L, VARONE F, BERGNA M, DE ANDRADE J, FALK J, HALLOWELL R, et al Pharmacological management of progressive-fibrosing interstitial lung diseases: a review of the current evidence. Eur Respir Rev 2018; 27 (150): 180074 doi: 10.1183/16000617.0074-2018.

49.- FLAHERTY KR, WELLS AU, COTTIN V, DEVARAJ A, WALSH SLF, INOUE Y, et al. Nintedanib in progressive fibrosing interstitial lung diseases. N Engl J Med 2019; 381 (18): 1718-27. doi: 10.1056/NEJMoa1908681.

50.- WELLS AU, FLAHERTY KR, BROWN KK, INOUE Y, DEVARAJ A, RICHELDI L, et al. Nintedanib in patients with progressive fibrosing interstitial lung diseases-subgroup analyses by interstitial lung disease diagnosis in the INBUILD trial: a randomised, double-blind, placebo-controlled, parallel-group trial. Lancet Respir Med 2020; 8 (5): 453-60. doi: 10.1016/ S2213-2600 (20) 30036-9.

51.- HERNÁNDEZ LÓPEZ JC, URCUQUI INCHIMA S. Activación y regulación del inflamasoma NLRP3 en las enfermedades infecciosas. Iatreia [Internet] 2012; 25 (4): 380-90. Disponible en: http://www. scielo.org.co/scielo.php?script=sci_arttext\&pid=S012107932012000400008\&lng=en.
Correspondencia a:

Dr. Julián Rondón-Carvajal

Medellín, Colombia

Email: julianrondoncarvajal@gmail.com 